\title{
A CELL-DEVS MODEL FOR LOGISTIC URBAN GROWTH
}

\author{
Bruno St-Aubin \\ Department of Systems and Computer Engineering \\ Carleton University \\ 1125 Colonel By Drive \\ Ottawa, ON, Canada \\ bruno.staubin@carleton.ca
}

\author{
Gabriel Wainer \\ Department of Systems and Computer Engineering \\ Carleton University \\ 1125 Colonel By Drive \\ Ottawa, ON, Canada \\ gwainer@sce.carleton.ca
}

\begin{abstract}
As society increasingly moves towards sustainable urban growth, urban planners and city officials require tools that efficiently support spatial decision-making. Modeling and simulation (M\&S) have been shown to be an adequate tool in this case. Cell-DEVS offers an optimized method of spatial simulation that can tackle computationally complex scenarios such as urban growth. To demonstrate this, we implement and discuss a well-known model, logistic urban growth (LUG), using the Cell-DEVS formalism. We show that this implementation supports the standard characteristics of LUG models by studying the variation of different parameters of the model such as restricted growth areas, spatial configuration and distance to features of interest.
\end{abstract}

Keywords: DEVS, Cell-DEVS, Visualization and Analysis, Logistic Urban growth.

\section{INTRODUCTION}

Earlier this year, the Population Division of the Department of Economic and Social Affairs of the United Nations published preliminary results of its Revision of World Urbanization Prospects. They report that, as of today, $55 \%$ of the world population lives in urban areas and the proportion is expected to rise to $68 \%$ by 2050 (United Nations 2018). The projected number for 2050 is 4 points higher than the one published in 2014 indicating a faster than anticipated global urbanization process (United Nations 2014). Furthermore, it found that $82 \%$ of North Americans, $81 \%$ of South Americans and $74 \%$ of Europeans now reside in urban areas. This report denotes, in an obvious manner, that sustainable urbanization should be a central goal for our society.

Modeling and simulation (M\&S) of urban growth patterns has shown to be an efficient tool to support decision-making for urban planners and city officials when planning future urban development. In this context, M\&S allows planners to quickly evaluate different scenarios at a low cost. Various approaches have been used to model urban growth, many of which rely on the cellular automata method (Shafia et al. 2018, Li et al. 2017, Ke et al. 2016). Unsurprisingly, a large portion of the research originates from China and India, the two countries expected to contribute the most to urban growth in the future.

Urban growth modeling is data intensive and involves complex dynamic processes; it can be computationally prohibitive. The Cell-DEVS (Discrete Event System Specification) approach offers a potential solution to this problem. In this paper, we show that Cell-DEVS can adequately represent urban growth by implementing a well-known method of urban growth modelling, logistic urban growth (LUG). We demonstrate that a Cell-DEVS model can consider restricted growth areas, distance to important geographic features, neighborhood urbanization and variations in spatial configuration. 


\section{BACKGROUND}

Cell-DEVS is an extension of DEVS that can be used for modeling and simulation of systems in a cell space. It is a combination of DEVS and cellular automata (CA) with explicit timing delays (Wainer 2009). Each cell becomes an individual atomic model and the cell space becomes a coupled model where all the cells are linked to their neighbors through input and output ports, as in regular DEVS models. Each cell receives $N$ inputs, usually from neighboring cells but they could also be provided by a regular DEVS model.

When a cell receives inputs, it triggers $\tau$, the local computing function that will determine the next state, $s^{\prime}$. At this moment, if the cell's future state $\mathrm{s}^{\prime}$ is different than its current state $\mathrm{s}$, it will schedule an output of its new state following a transportation delay specified by $d$. Whenever a cell changes state, its new state s' and scheduled time for transition are added to a local queue. Cells with transport delays will always output their new state, provided a state change has occurred. If a cell's state does not change following the local computing function $\tau$, it becomes passive and waits for further external events.

Cells can also be implemented using inertial delays which allow pre-emption of the cell's state transition. A cell with inertial delay that does not manage to keep its new state until the next scheduled time elapses is pre-empted and foregoes its output phase for the pre-empted state transition. The inner workings of a cell with inertial delay are slightly different than one with transport delays, interested readers should refer to (Wainer 2009) for a more thorough explanation.

Urban growth modeling and simulation has generated large amounts of research in the past few years. Researchers have employed numerous approaches to simulate the spread of urbanization across space and time. Artificial Neural Networks (ANN) based methods have been in use for at least 15 years (Pijanowski et al. 2002, Yeh, Xia, 2004) but seem to be experiencing a resurgence in popularity in the past few years. For example, Pijanowski et al used an ANN based land transformation model (Pijanowski et al. 2014) within a geographic information system (GIS) environment to simulate urban growth. Grekousis et al. also used ANN combined with fuzzy set logic within a GIS environment to simulate urban growth in the Athens area. (Grekousis et al., 2013)

However, the bulk of urban growth modelling and simulation is accomplished by combining the cellular automata (CA) approach with other modelling techniques. CA is generally accepted as the most efficient method to model and simulate urban growth due to its simplicity, adaptability and for its ease of integration with other modeling techniques (Aburas et al. 2016, Santé et al. 2009, 2010). In their extensive reviews of urban growth CA research, Aburas et al, as well as Santé et al identify a very large number of CA based techniques and discuss their strong points and weaknesses. Detailing all of them, in the scope of this paper, would be a redundant exercise. Instead, we will only present some of the more notable examples.

In recent research, the most widespread urban growth CA methods are based on Logistic Regression (LR), Markov Chains (MC), SLEUTH and Fuzzy Logic. In LR based CA models, the logistic equation drives the urbanization process and the coefficients within the equation are obtained by regression. Since it is based on a mathematical equation, LR CA has difficulty considering the complex relationships in an urban growth scenario. Nonetheless, it has been used frequently in the past few years and improvements to the model are consistently being put forth (Shu et al. 2017, Feng 2017, Li et al. 2017). MC-CA models predict the future urbanization state of a cell-space by analyzing its urbanization state from previous iterations using stochastic processes. This approach can be used with limited datasets but fails to consider socio-economic factors (Al-Sharif, Pradhan 2013). SLEUTH based CA methods take their name from the parameters they consider namely, slope, land use, exclusion, urban extent, transportation, and hill shade. SLEUTH has some issues that are well documented by its inventor (Clark 2008) but it has been used to simulate urban growth for the past 20 years (Chaudhuri, Clarke, 2014). Methods based on Fuzzy CA involve clustering space using 
fuzzy logic (Grekousis et al., 2013). This allows one element to belong to multiple clusters to a certain degree, facilitating complex analysis. The main advantages of Fuzzy CA is that it considers statistical correlations between predictor factors and it supports large, complex data sets (Aburas et al. 2016).

A common approach to simulate urban growth over time and space is to use the logistic cellular automata (LCA) model. The transition rules for this model are based on variations of the logistic equation developed by Pierre-François Verhulst in the early $19^{\text {th }}$ century (Verhulst, 1845, Brauer, Castillo-Chàvez, 2001). Briefly put, the equation states that population growth, unimpeded by external factors, will follow an exponential curve that will diminish as population grows and completely stop at population maturity. This equation is used in a large range of fields including chemistry, ecology, demography, economics, etc.

Feng Y. and Shu B. \& al. have proposed models that expand the logistic equation in similar ways (Feng, 2017, Shu \& Al. 2017). In both cases, the models consider a Moore neighborhood of variable size ( 3 X 3 , $5 \times 5$ or $7 \times 7$ ), the cells have a binary value ( 0 for non-urban, 1 for urban), and some cells are restricted. The probability that a non-urban cell transitions to an urban cell is defined using the following equation:

$$
P_{i j}^{t}=P_{d i} \times P_{N i, t} \times \text { Con }
$$

Where

$$
\begin{array}{cl}
P_{i j}^{t} & \text { Probability of transition for cell } \mathrm{i}, \mathrm{j} \text { at time t } \\
P_{d i} & \text { Probability of transition contributed by distance to geographical features } \\
P_{N i, t} & \text { Probability of transition due to urban neighbors } \\
\text { Con } & \begin{array}{l}
\text { Represents physical constraints for urbanization (i.e. water, canyon, } \\
\text { protected zones, etc. }
\end{array}
\end{array}
$$

This equation can be expanded to the following:

$$
P_{i j}^{t}=\frac{1}{1+e^{-\left(a_{0}+\sum_{n=1}^{m} a_{n} x_{n, i j}\right)}} \times \frac{\sum_{x, y=1}^{x, y=n} \operatorname{con}\left(S_{x y}=\text { urban }\right)}{n^{2}-1} \times \operatorname{Bin}\left(\text { cell }_{i j} \neq \text { restricted }\right)
$$

Where

$$
\begin{array}{cl}
a_{0} & \text { A constant } \\
a_{1}, a_{2} \ldots a_{m} & \text { Regression coefficients for distance factors } \\
x_{n, i j} & \text { Normalized distance to geographical feature } \\
\text { con } & \text { A condition that returns } 1 \text { if the } \mathrm{x}, \mathrm{y} \text { cell is urbanized. } \\
\text { Bin } & \text { A binary function that returns } 1 \text { if the cell is not restricted }
\end{array}
$$

In (2), the regression coefficients $a_{1}, a_{2} \ldots a_{m}$ are obtained by calibrating the model using existing data and a regression technique. Furthermore, some authors advocate adding a stochastic disturbance to this equation (Feng 2017, White et al. 1993). This provided interesting analysis opportunities, so it was also considered in this experimentation.

$$
R=1+(-\ln r)^{a}
$$

where

$R$ Stochastic disturbance applied to the probability of transition

$r \quad$ Random real number between 0 and 1

$a$ A parameter ( 0 to 10 ) that adjusts the effect of the stochastic factor 
Considering the stochastic disturbance, the probability of transition becomes:

$$
P_{i j}^{t}=\frac{1}{1+e^{-\left(a_{0}+\sum_{n=1}^{m} a_{n} x_{n, i j}\right)}} \times \frac{\sum_{x, y=1}^{x, y=n} \operatorname{con}\left(S_{x y}=\text { urban }\right)}{n^{2}-1} \times \operatorname{Bin}\left(\text { cell }_{i j} \neq \text { restricted }\right) \times\left(1+(-\ln r)^{a}\right)
$$

With the objective of modeling urban growth using a Cell-DEVS approach, we opted to use the Logistic CA approach due to its simplicity to implement and its widespread use in urban growth CA research. The goal is to demonstrate that the discrete event specification is a suitable technique to model and simulate this phenomenon.

\section{LOGISTIC URBAN GROWTH (LUG) AS A CELL-DEVS MODEL}

To demonstrate the application of the formalism to this model, we used a basic mock map that provided geospatial features of interest. This was done to avoid the otherwise complex and time-consuming image analysis pre-processing required to obtain the initial values for the cell-space. In order to deal with real source maps, we need to consider many intricacies that are outside the scope of this research. For example, anti-aliasing makes it difficult to automatically separate these images into well-defined initial states. Another example is the complexity of the geospatial data: hydrography, road networks, buildings, etc.

The regression coefficients were not calibrated from training data. In a real-world scenario, regressing the coefficients would involve comparing the real future state of the cell-space with the simulator generated future state of the cell-space, modifying the coefficients and iterating in this manner until satisfactory coefficients were found. Again, we employed this simplification to avoid a time-consuming process that is outside the scope of this research. Instead, the coefficients were varied methodically so that their impact on the simulation could be studied through visualization and analysis tools.

The final assumption we adopted was that the spatial configuration of the geographical map was static across the time span of the simulation. In real-world scenarios, it would be possible for the configuration to evolve, for example, a new road could be constructed mid-simulation. This assumption allowed us to pre-process the first factor in the LUG equation (4) prior to running the simulation and save it on a separate layer where it could easily be retrieved by the simulation engine at runtime.

\subsection{The formal DEVS model}

To execute the simulation, we employed $\mathrm{CD}++$, a mature simulation engine specialized in DEVS simulation on cell-spaces. Additionally, we used the Cell-DEVS WebViewer, a lightweight, web-based platform to visualize the outputs of the simulation in video form (St-Aubin, Hesham, Wainer, 2018). The formal CellDEVS model for the simulation studied is presented in figure 1 below.

\section{Model parameters}

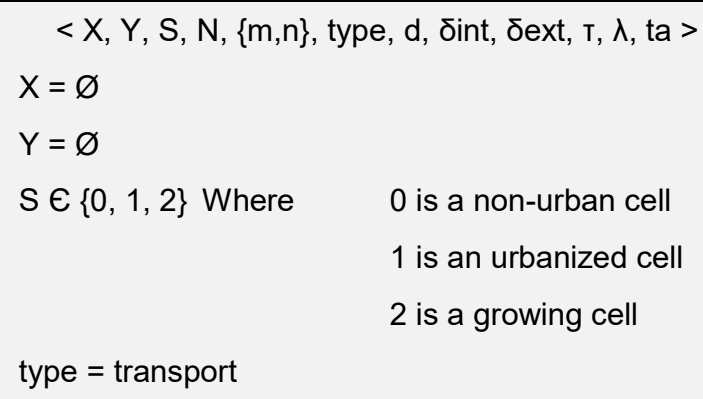

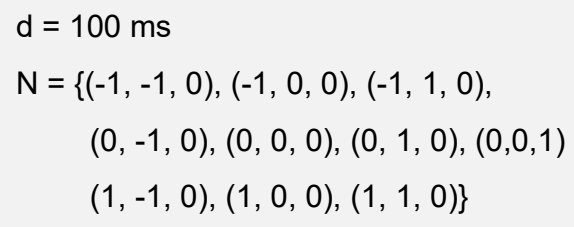

$\mathrm{T}: \mathrm{N} \rightarrow \mathrm{S}$ Where $\mathrm{S}$ are the rules for cell state transition defined in the table to the right

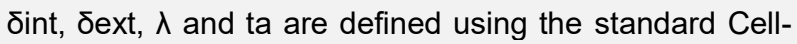
Devs specifications. 
Transition rules

\begin{tabular}{|l|l|}
\hline unchanged & Any value from the $P_{\text {di }}$ layer \\
\hline 2 & $(0,0,0)=0$ AND Probability Threshold is met \\
\hline 1 & $(0,0,0)=2$ \\
\hline 2 & $(0,0,0)=1$ AND Number Urban neighbors $<9$ \\
\hline
\end{tabular}

Figure 1: The formalized LUG model for Cell-DEVS Implementation of the model.

The first step to prepare the simulation consists of designing a mock map suited to run the simulation and convert it to a file of initial states for the simulation. To do so, a map with a restricted set of geospatial features was designed (See figure 2) using vector geometry, then converted to a raster format. For this experiment, hydrography, road networks and attracting point features were considered. In the map, attracting features were labelled in a generic manner. In a real-world scenario, these features could be, for example, urban centers, bus stations, commercial centers, learning institutions, etc. Each geospatial feature is associated to a cell-state according to its color, as defined in figure 2. Obtaining the initial states for the simulation cell-space is, therefore, a matter of associating each pixel of the raster map to a cell-state.

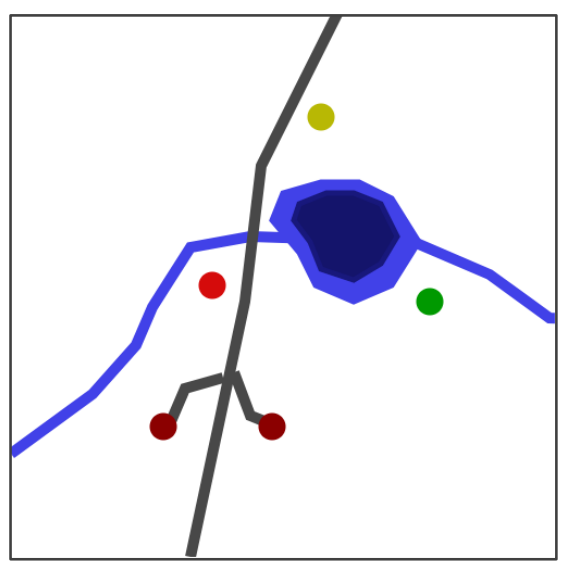

\begin{tabular}{|lcl|}
\hline Color & Value & Feature name \\
\hline & 10 & Line Feature \\
\hline & 20 & Water \\
\hline & 99 & Water restricted \\
\hline & 30 & Point Feature A \\
\hline & 40 & Point Feature B \\
\hline & 50 & Point Feature C \\
\hline & 60 & Point Features D \\
\hline & 100 & Non-urbanized cell \\
\hline & 20 & Urbanized cell \\
\hline
\end{tabular}

Figure 2: Sample geospatial map with a legend showing the values associated to each color.

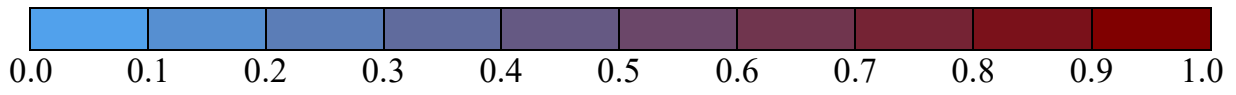

Figure 3: The gradient for the $P_{d i}$ layer.

The second step consists of computing the $P_{d i}$ factor for each cell. As mentioned before, for a given cell, we assumed that the spatial configuration remains static across the simulation. As such, the $P_{d i}$ factor is also static, and its value can be pre-processed and stored in an additional layer, the $P_{d i}$ layer, that is overlaid on the main $2 \mathrm{D}$ cell space. $P_{d i}$ values are can go from 0 , the lowest probability of transition, to 1 , the highest probability of transition. Therefore, the layer can be visually represented using the gradient in figure 3 .

The third and final step consists of generating a set of urbanized cells to trigger the cell transition process at simulation start up. Different configurations were tried (single random cells, cells in a cross layout and clusters), and clusters provided the most organic experience. Single cells had more difficulty triggering initial transitions because they lack other urbanized neighbors which has a significant impact on the transition function. Non-urbanized cells were given the value of 100 while urbanized cells were given the value of 101.0 and 1 were avoided because these values were used by the $P_{d i}$ layer. 
It should be noted that non-restricted cells can be urbanized. This allows the urbanization process to cross roads, rivers and other features and retains the geospatial configuration information for visualization purposes. Urban cells that contain a feature will show as a darkened version of the original cell color and will have a value that is 1 higher than its non-urban state. Each cell also has an extra state that represents it growing. A cell will alternate between growing and urbanized until all neighbors are either urbanized or restricted. Once all its neighbors are urbanized, the cell will settle on the urbanized state. By alternating states, we guarantee that a cluster cannot stop growing if it doesn't acquire a new cell.

\subsection{LUG Model parameter study}

To evaluate the effect of each parameter on the results, we systematically modify one parameter while keeping the others constant. In most cases, we started by a random selection of parameters, observed the outputs, then modified them according to the parameter under study. The experimentation was more extensive than what is shown in this section but, for the sake of brevity, only the more interesting cases will be discussed. The parameters used will be provided for result reproduction purposes.

The probability of transition threshold defines the value beyond which the transition equation (4) will cause a cell to become urbanized. It has an immediate and obvious impact on the transition process as can be seen in the following images. For each of these cases, the simulation was run using the same coefficients while varying the threshold only.

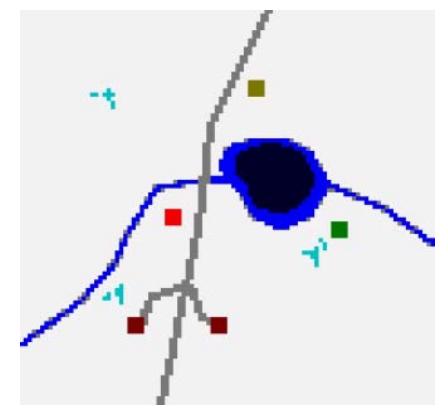

Initial State

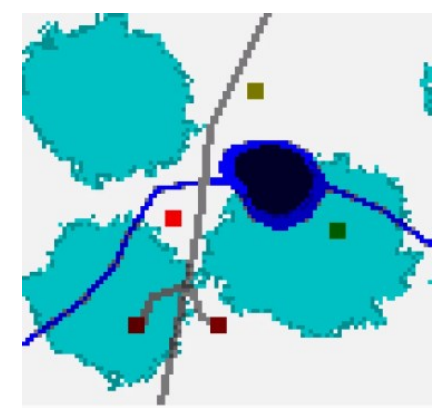

Threshold 0.9

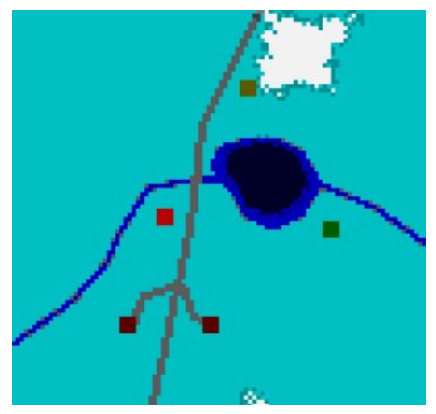

Threshold 0.5

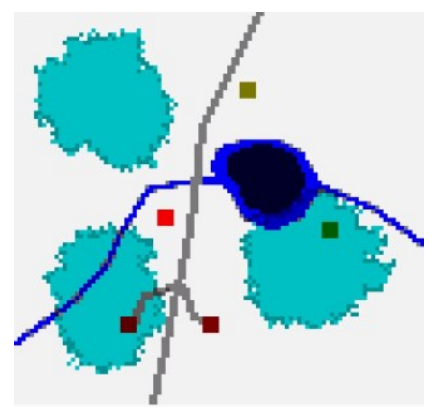

Threshold 1.1

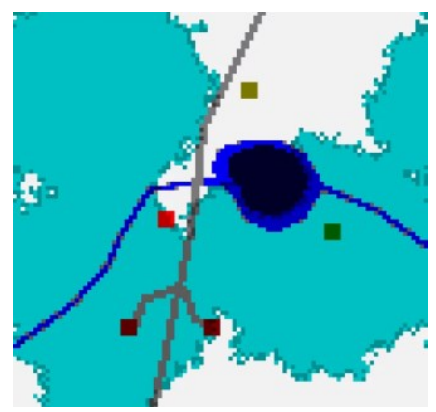

Threshold 0.7

Figure 4: Variation of the probability of transition threshold. Top-left frame shows the initial state of the cell-space. Each following frame shows the final state of the cell-space for a given threshold.

None of the threshold variation simulation were able to completely urbanize the cell space. However, as the threshold increases, the final clusters diminish in size and the total urbanized area is reduced. Since the probability equation (4) result must exceed higher thresholds, it is more difficult for the cells to transition to an urban state. Note that the effect of restricted cells can already be observed from figures 4 and 5 . 
The impact of the stochastic disturbance is subtler than the threshold variation. It introduces uncertainty in the cell transition process; running two simulations with the same parameters will yield different outcomes. It should also be noted that without this factor, for a given simulation, it would be possible for some cells to never be able to urbanize. To study the stochastic disturbance, the same distance coefficients as before were used, a threshold variation of 0.9 was used and the simulation was run twice.
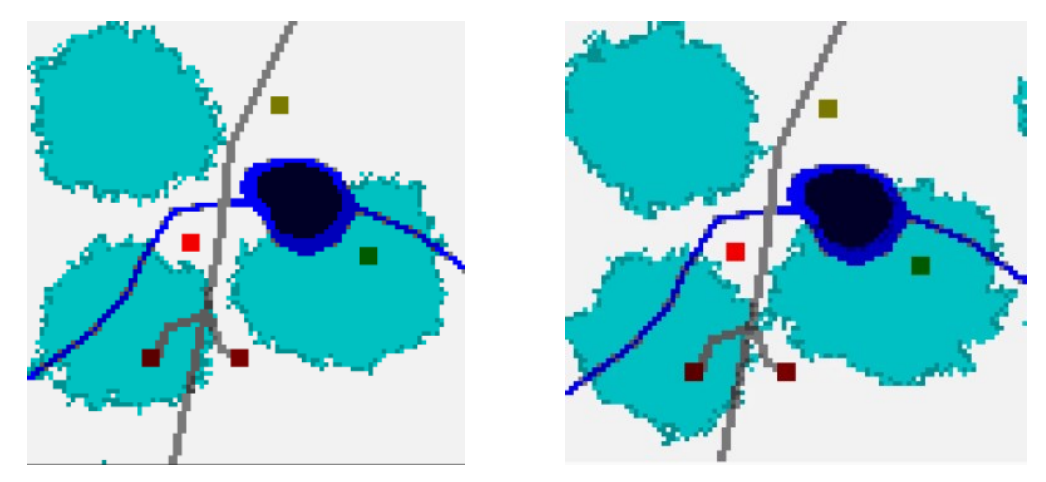

Figure 5: Two simulation run with the same parameters will generate different results.

In the example above, the difference between both simulation results can be observed. For example, the two bottom clusters have begun to merge on the right while they are still distinct on the left. We can also see that the simulation on the right has begun wrapping around the cell space while the one the left has not. Let us note that wrapping around the cell-space, for an urban growth model, does not reflect reality properly. Considering our goal to show that DEVS, as a methodology, can represent urban growth, we adopted this simplification to facilitate the definition of transition rules at the edges of the cell-space.

The distance coefficients, in the LUG equation (4), contribute to the urbanization potential of cells near geospatial features. Cells nearer to a feature with a high coefficient will have a higher urbanization potential. As the distance between a cell and the point feature increases, the contribution to the urbanization potential of the cell decreases proportionally. In both examples below, we can clearly see that the urbanization process is skewed towards the point features with more extreme coefficient values. This is made obvious by their corresponding $\mathrm{P}_{\mathrm{di}}$ layers. As the urbanized clusters expand outside of the higher weight zones, the transition process slows down dramatically and, in both cases the cell space is never entirely urbanized.
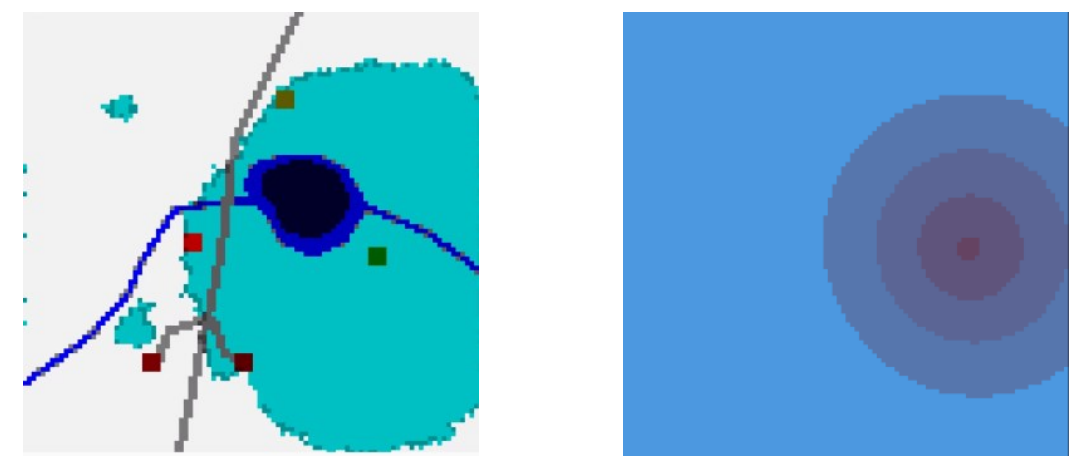

$$
\begin{aligned}
\text { Coefficients } \\
C=0 \\
D_{A}=-0.016 \\
D_{B}=-4.237 \\
D_{C}=0.043 \\
D_{D}=0.0275 \\
D_{\text {Line }}=-0.142
\end{aligned}
$$

Figure 6: Attraction towards the point feature B (green). 

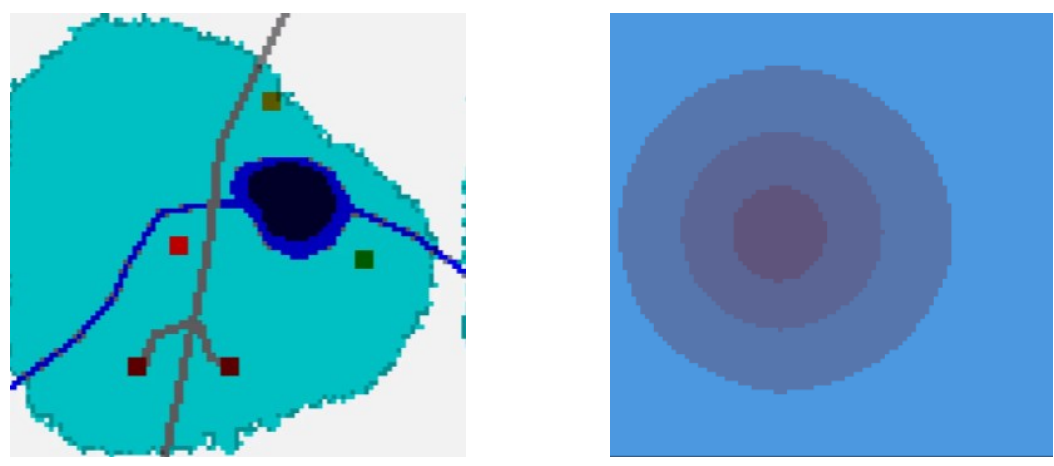

$$
\begin{aligned}
\text { Coefficients } \\
C=0 \\
D_{A}=-3.016 \\
D_{B}=-0.237 \\
D_{C}=0.043 \\
D_{D}=0.0275 \\
D_{\text {Line }}=-0.142
\end{aligned}
$$

Figure 7: Attraction towards the point feature A (bright red).

The impact of increasing the attraction towards linear features was more difficult to analyze. It was expected that the $P_{d i}$ layer would show a distribution parallel to the linear feature representing the road (in gray), this is not the case here. This could betray an underlying issue with the function used to calculate the distance between a cell and a line. However, it can still be seen that a linear feature crossing the whole cell-space has a massive impact on the $P_{d i}$ layer which shows much higher values across the whole cell-space. Accordingly, this simulation managed to urbanize the whole cell-space in about half the time of the simulations discussed before.
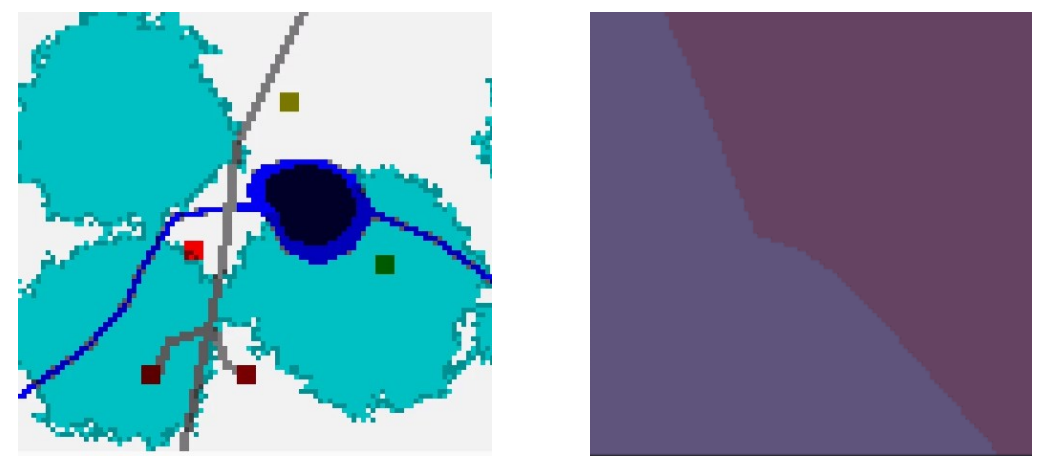

$$
\begin{aligned}
\text { Coefficients } \\
C=0 \\
D_{A}=-0.016 \\
D_{B}=-0.237 \\
D_{C}=0.043 \\
D_{D}=0.0275 \\
D_{\text {Line }}=-2.142
\end{aligned}
$$

Figure 8: Attraction towards linear feature. This simulation urbanized the whole cell space. The image is taken halfway through the simulation.

The case study below combines a high attraction towards point feature B (green) and a medium attraction towards point features D (dark red). The coefficient for the linear feature is also high and positive which means that cells will have more difficulty transitioning to an urban state when they are near the line. The top left cluster mostly stagnated because it has no nearby attracting features and the linear feature acts as a repulsive barrier for its expansion. The bottom right cluster expanded greatly because it is very close to point feature B (green). It also shows the pull generated by both point features D (dark red). Finally, the animated visualization shows that once outside the darker areas of the $P_{d i}$ layer, cells have much more difficulty transitioning. 

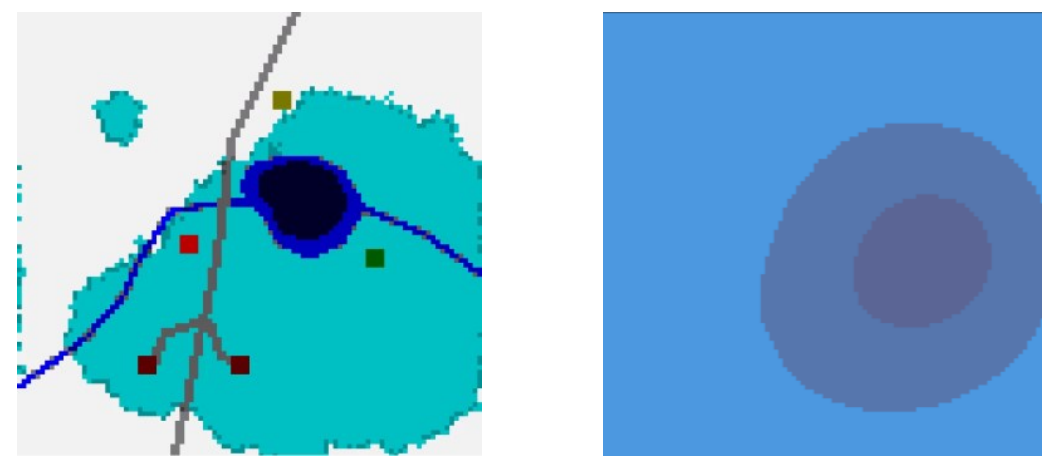

$$
\begin{aligned}
\text { Coefficients } \\
C=0 \\
D_{A}=-0.016 \\
D_{B}=-2.437 \\
D_{C}=-0.243 \\
D_{D}=-0.975 \\
D_{\text {Line }}=1.642
\end{aligned}
$$

Figure 9: A combination of coefficients where point feature B has high attraction, point features $\mathrm{D}$ have medium attraction and the line feature has high repulsion.

We also ran different scenarios combining many coefficient variations, the impact of which can be distinctly observed in the results, albeit with some difficulty. For example, the $P_{d i}$ layer shows a definite high attraction area delimited by the point features $\mathrm{A}, \mathrm{B}$ and $\mathrm{C}$ with the innermost ring showing a tendency towards point feature A. The repulsive effects of point features D can be clearly seen in the simulation visualization on the left. Again, the line feature is more difficult to observe but it does seem to flatten the $P_{d i}$ rings on the left side. Furthermore, the lingering effect of the restricted cells (dark blue) is also seen on the visualization on the left. Indeed, as cells are growing around the lake, the urban area becomes more asymmetric and diverges from the $P_{d i}$ layer.
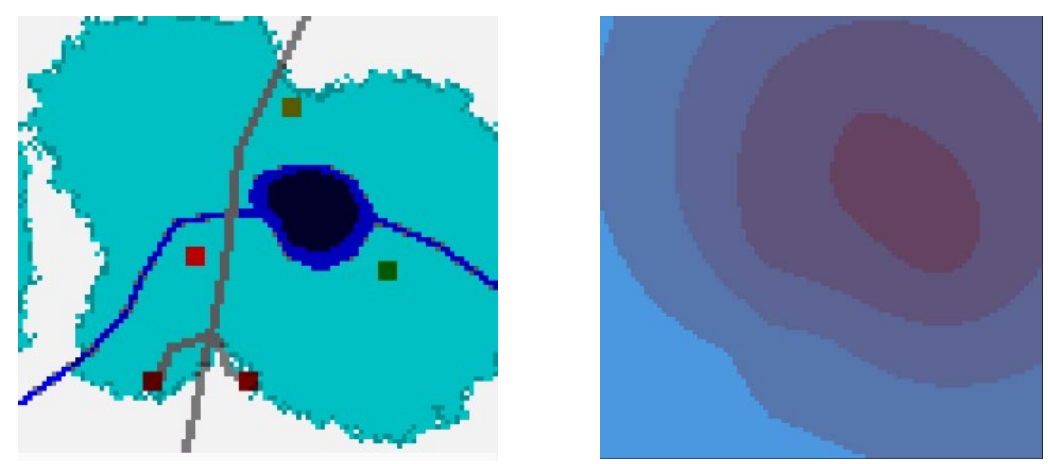

$$
\begin{aligned}
\text { Coefficients } \\
\mathrm{C}=0.040 \\
\mathrm{D}_{\mathrm{A}}=-1.016 \\
\mathrm{D}_{\mathrm{B}}=-1.837 \\
\mathrm{D}_{\mathrm{C}}=-0.643 \\
\mathrm{D}_{\mathrm{D}}=1.275 \\
\mathrm{D}_{\text {Line }}=1.642
\end{aligned}
$$

Figure 10: A combination of coefficients where each feature has a distinct impact on the simulation. Image taken at about $1 / 3$ of the simulation length.

To analyze the effect of varying the spatial configuration of the initial mock map, two additional mock maps were built, and the coefficients discussed in the preceding section were tested against them. From figure 11 presented below, the impact that a different spatial configuration has on the $P_{d i}$ layer and the simulation itself can be observed. The coefficients favor growth towards the point features A, B and C while point features $\mathrm{D}$ and the line feature have a repulsive effect on urbanization. The repulsive effect is more obvious in the right-most simulation result. The effect of a larger cluster of restricted cells (dark blue) is more noticeable in the center map, the result being a highly asymmetrical urban area. 

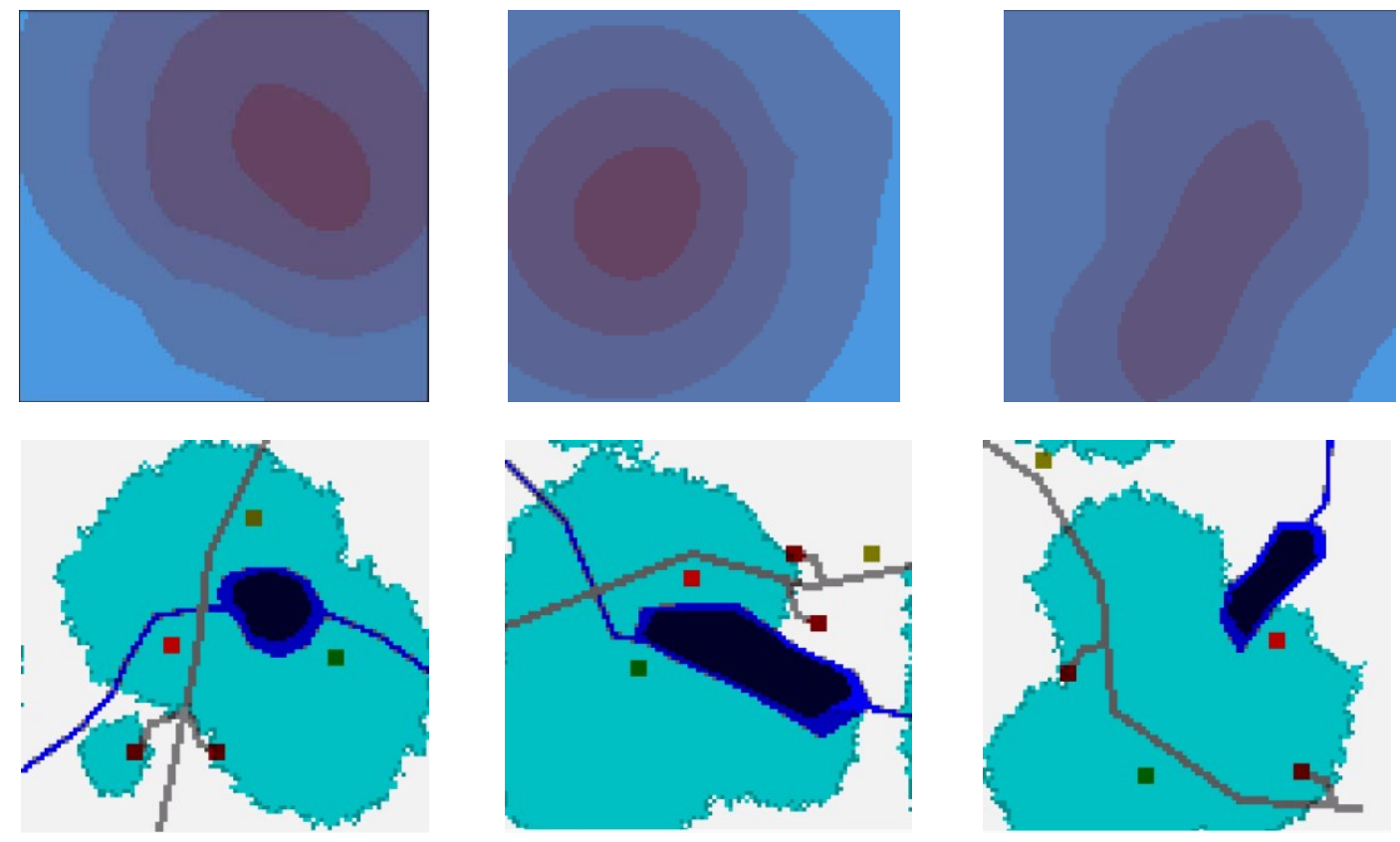

Figure 11: Variation on the spatial configuration of the cell space, using coefficients from Figure 10. All images taken at about $1 / 3$ of the simulation length.

\section{CONCLUSION}

We presented a Cell-DEVS definition and an implementation of the Logistic Urban Growth model using Cell-DEVS, a DEVS based cellular automata formalism. Although Cell-DEVS has been used to model and simulate real-world systems in various scenarios, it had yet to be employed to model urban growth. The research presented in this paper filled this gap. Since the objective of this paper was to show that urban growth could be modeled using a DEVS based methodology, we chose to implement one of the simpler CA-based urban growth models based on the logistic equation. The results presented here show that CellDEVS adequately supports the Logistic Urban Growth model.

Future work on this model should be focused on using real-world data to generate the initial values and state of the cell-space. This would provide the data required to verify, validate and likely further improve the model. Using real data also implies a calibration phase where the distance coefficients of the logistic equation could be obtained from training data using logistic regression or other regression techniques. This would be an essential step in the verification and validation of the model as well. Another avenue of research would be to altogether implement other popular models of CA based urban growth such as the SLEUTH approach or the Markov-CA model. This would provide the opportunity to compare results and performance for different approaches. Considering that urban spaces are dynamic and evolutive, adding the capability to consider geospatial configuration changes in the map would lead to more realistic simulations of urban growth using this model. Integrating aspects of other models, particularly the SLEUTH approach, could also lead to more simulation results that are more faithful to the real-world.

Urban Growth modeling is becoming an invaluable tool for urban planners, municipal government officials and other decision makers. Generic modeling tools such as DEVS based tools are uniquely positioned to provide a flexible and intuitive process to generate reusable and easy to validate models and simulations. Through its hierarchical and modular approach, its decoupling of the models and the simulator, DEVS could act as a generic platform for users to model a variety of phenomenon involved in city planning such as 
vehicle traffic, pedestrian behavior, pollution dispersions and, as this research as shown, urban growth. The complexity of modeling and simulation is often prohibitive for non-expert users. Increased ease-of-use and adaptability to different scenarios is one way to work around this issue and provide decision makers with more varied and better decision-making tools.

\section{REFERENCES}

Aburas, M. M., Ho, Y. M. Ramli, M. F. Ash'aari, Z. H., "The simulation and prediction of spatio-temporal urban growth trends using cellular automata models: A review," Int. J. Appl. Earth Obs. Geoinf., vol. 52, pp. 380-389, 2016.

Al-Sharif, A.A., Pradhan, B., "Monitoring and predicting land use change in Tripoli Metropolitan City using an integrated Markov chain and cellular automata models in GIS", Arabian J. Geosci., 1-11, 2013

Brauer F., Castillo-Chàvez C., "Mathematical Models in Population Biology and Epidemiology," Texts in Applied Mathematics, Vol. 40, Springer, 416 p., 2001

Chaudhuri, G. Clarke, K. C., "The SLEUTH land use change model : A review," Environ. Resour. Res., vol. 1, no. 1, pp. 88-105, 2014.

Clarke, K. C., "A Decade of Cellular Urban Modeling with SLEUTH: Unresolved Issues and Problems," Ch. 3 in Planning Support Systems for Cities and Regions, R. K. Brail, Ed. Cambridge, MA, pp. 4760, 2008.

Feng Y., "Modeling Dynamic Urban Land-Use Change with Geographical Cellular Automata and Generalized Pattern Search-Optimized Rules," International Journal of Geographical Information Science, Vol. 31, No. 6, Taylor \& Francis Group, pp. 1198-1219, 2017

Grekousis, G., Manetos, P., Photis, Y.N., "Modeling urban evolution using neural networks: fuzzy logic and GIS: the case of the Athens metropolitan area". Cities 30, pp. 193-203, 2013.

Ke, X., Qi, L., Zeng, C., "A partitioned and asynchronous cellular automata model for urban growth simulation," Int. J. Geogr. Inf. Sci., vol. 30, no. 4, pp. 637-659, 2016.

Li, X., Gong, P., Yu, L., Hu, T., “A segment derived patch-based logistic cellular automata for urban growth modeling with heuristic rules," Comput. Environ. Urban Syst., vol. 65, pp. 140-149, 2017.

Li, X., Lu, H., Zhou, Y., Hu, T., Liang, L., Liu, X., Hu, G., Yu, L., "Exploring the performance of spatio-temporal assimilation in an urban cellular automata model," Int. J. Geogr. Inf. Sci., vol. 31, no. 11, pp. 2195-2215, 2017.

Pijanowski, B.C., Brown, D.G., Shellito, B.A., Manik, G.A., "Using neural networks and GIS to forecast land use changes: a land transformation model”, Computers. Environ. Urban Syst., 26 (6), pp. 553$575,2002$.

Pijanowski, B.C., Tayyebi, A., Doucette, J., Pekin, B.K., Braun, D., Plourde, J. "A big data urban growth simulation at a national scale: configuring the GIS and neural network based Land Transformation Model to run in a High Performance Computing (HPC) environment”. Environ. Model. Softw. 51, pp. 250-268, 2014. 
Santé, I., García, A. M., Miranda, D., Crecente, R., "Analysis of Operational Urban Cellular Automata Models Other Methods None," Proc. 2nd WSEAS Int. Conf. URBAN Plan. Transp., pp. 91-98, 2009.

Santé, I., García, A. M., Miranda, D., Crecente, R., "Cellular automata models for the simulation of realworld urban processes: A review and analysis," Landsc. Urban Plan., vol. 96, no. 2, pp. 108-122, 2010 .

Shafia, A., Gaurav, S. Bharath, H. A., "Urban growth modelling using Cellular Automata coupled with land cover indices for Kolkata Metropolitan region," IOP Conf. Ser. Earth Environ. Sci., vol. 169, no. 1, 2018.

Shu B., Bakker M.M., Zhang H., Li Y., Qin W., Carsjens G.J, "Modeling Urban Expansion by Using Variable Weights Logistics Cellular Automata: A Case Study of Nanjing China," International Journal of Geographical Information Science, Vol. 31, No. 7, Taylor \& Francis Group, pp. 1314-1333, 2017

St-Aubin B., Hesham O., Wainer G., "A Cell-DEVS Visualization and Analysis Platform”, Proceedings of the 50th Computer Simulation Conference, SummerSim-SCSC, 12 p.

United Nations, 2018, “World Urbanization Prospects 2018: Key Facts”, https://population.un.org/wup/ Publications/, accessed on Dec. 16, 2018.

United Nations, 2014, "World Urbanization Trends 2014: Key Facts", https:/population.un.org/wup/ Publications/, accessed on Dec. 16, 2018.

Verhulst P-F., "Recherches mathématiques sur la loi d'accroissement de la population », Nouveaux Mémoires de l'Académie Royale des Sciences et Belles-Lettres de Bruxelles, 1845

Wainer, G.A, 2009, "Discrete-event modeling and simulation: a practitioner's approach," CRC Press, Taylor \& Francis Group, 485 p.

White, R., Engelen, G., "Cellular Automata and Fractal Urban Form: A Cellular Modelling Approach to the Evolution of Urban Land-Use Patterns," Environ. Plan. A, vol. 25, no. 8, pp. 1175-1199, 1993.

Yeh, A., Xia, L, "Integration of neural networks and cellular automata for urban planning." Geospat. Info. Sci. 7 (1), pp. 6-13, 2004

\section{AUTHOR BIOGRAPHIES}

BRUNO ST-AUBIN. Is pursuing a $\mathrm{PhD}$ in Electrical and Computer Engineering at Carleton University where he researches web-based simulation and visualization. His email address is bruno.staubin@carleton.ca.

GABRIEL WAINER is a Professor at the Department of Systems and Computer Engineering at Carleton University. He is a Fellow of the Society for Modeling and Simulation International (SCS). His email address is gwainer@sce.carleton.ca. 